\title{
A Computational Model of the Epidermis With the Deformable Dermis and Its Application to Skin Diseases
}

Kota Ohno

Hokkaido University

Yasuaki Kobayashi

Hokkaido University

Masaaki Uesaka

The University of Tokyo

Takeshi Gotoda

Nagoya University

Mitsuhiro Denda

Shiseido Global Innovation Center

Hideyuki Kosumi

Hokkaido University

Mika Watanabe

Hokkaido University

Ken Natsuga

Hokkaido University

Masaharu Nagayama ( $\sim$ nagayama@es.hokudai.ac.jp )

Hokkaido University

\section{Research Article}

Keywords: computational model, epidermis, skin, homeostasis

Posted Date: March 23rd, 2021

DOl: https://doi.org/10.21203/rs.3.rs-352112/v1

License: (c) (i) This work is licensed under a Creative Commons Attribution 4.0 International License.

Read Full License 


\title{
A computational model of the epidermis with the deformable dermis and its application to skin diseases
}

\author{
Kota Ohno ${ }^{1, *}$, Yasuaki Kobayashi ${ }^{1, *}$, Masaaki Uesaka $^{2}$, Takeshi Gotoda ${ }^{3}$, Mitsuhiro \\ Denda $^{4}$, Hideyuki Kosumi ${ }^{5}$, Mika Watanabe ${ }^{5}$, Ken Natsuga ${ }^{5}$, and Masaharu Nagayama ${ }^{1, * *}$ \\ ${ }^{1}$ Research Center of Mathematics for Social Creativity, Research Institute for Electronic Science, Hokkaido \\ University, Sapporo, Japan \\ ${ }^{2}$ Graduate School of Mathematical Sciences, The University of Tokyo, Tokyo, Japan \\ ${ }^{3}$ Graduate School of Mathematics, Nagoya University, Nagoya, Japan \\ ${ }^{4}$ Shiseido Global Innovation Center, Yokohama, Japan \\ ${ }^{5}$ Department of Dermatology, Hokkaido University Faculty of Medicine and Graduate School of Medicine, \\ Sapporo, Japan \\ *These authors equally contributed to this work \\ ** Corresponding author: nagayama@es.hokudai.ac.jp
}

\begin{abstract}
The skin barrier is provided by the organized multi-layer structure of epidermal cells, which is dynamically maintained by a continuous supply of cells from the basal layer. The epidermal homeostasis can be disrupted by various skin diseases, which often cause morphological changes not only in the epidermis but in the dermis. We present a three-dimensional agent-based computational model of the epidermis that takes into account the deformability of the dermis. Our model can produce a stable epidermal structure with well-organized layers. We show that its stability depends on the cell supply rate from the basal layer. Modeling the morphological change of the dermis also enables us to investigate how the stiffness of the dermis affects the structure and barrier functions of the epidermis. Besides, we show that our model can simulate the formation of a corn (clavus) by assuming hyperproliferation and rapid differentiation. We also provide experimental data for human corn, which supports the model assumptions and the simulation result.
\end{abstract}

\section{Introduction}

Skin is a pivotal organ that prevents water loss and protects us from various kinds of external pathogens and stimuli $^{1,2}$. Stem cells in the basal layer continuously supply cells into suprabasal layers, which consist of the spinous, granular, and cornified layers from below. The cornified layer, the outermost part of the skin, consists of flat, regularly stacked cornified cells and the lipids filling the spaces between them, and its organized structure is responsible for epidermal barrier functions. To understand epidermal homeostasis and its barrier function, it is crucial to elucidate the mechanism of how the organized layer structure is maintained.

Mathematical modeling is a useful tool to investigate the emergence of epidermal homeostasis as a complex phenomenon. Among others, agent-based models have been widely adopted to study homeostatic properties of the epidermis $^{3-6}$. One advantage of using agent-based models is that it can easily incorporate various features, such as stem cell dynamics, differentiation, lipid production and secretion, and cell morphology. Pathological states of the skin can also be easily created, which enables one to study wound healing ${ }^{7-9}$ and development of psoriasis ${ }^{10}$. An integrated model of the epidermis that includes all features relevant to epidermal homeostasis would be desired to simulate various skin diseases and understand their mechanisms.

One factor that needs to be taken into account to have such an integrated model is a localized layer of calcium ions beneath the cornified layer, which could affect barrier functions ${ }^{11-18}$. Mathematical models were proposed for the calcium gradient in the epidermis ${ }^{19,20}$, as well as localized calcium excitation in cultured keratinocytes ${ }^{21}$, and 
the effect of the calcium layer on the epidermal structure was investigated ${ }^{22,23}$. The effect of calcium was further studied by using agent-based models ${ }^{24,25}$. By introducing an agent-based model of the epidermis, we suggested that the acceleration of differentiation due to calcium ions could stabilize the structure of the boundary between the granular layer and the cornified layer ${ }^{26}$.

Another important factor is the shape of the dermis, which could affect the spatial patterns of cell supply from the basal layer. The effect of dermal shape on the thickness of the epidermis was studied using a rigid, undulating dermis ${ }^{27}$, which suggested that an increase of the surface area due to dermal undulations could lead to an increase of epidermal thickness; This effect has been supported by an experiment ${ }^{28}$. Dynamical processes of dermal deformation were also studied: We proposed an agent-based model with the deformable dermis, which successfully simulated upward protrusions of the dermis starting from a flat dermis ${ }^{29}$, as observed in real human epidermis. It is well known that growing tissues can develop a spatial structure due to the buckling instability ${ }^{30-38}$; our model has revealed that the structure could also affect the spatial patterning of stem cells.

Our two previous models mentioned above are complementary: the epidermal model ${ }^{26}$ has not taken into account deformability of the dermis; The dermal deformation mode ${ }^{29}$ has taken into account only the dermis, the basement membrane, and the basal layer, disregarding the suprabasal layers. In this work, we integrate these two models into a unified model that can simulate epidermal homeostasis with the deformable dermis. This model includes important aspects for simulating the maintenance of the epidermis, such as cell division in the basal layer, calcium-dependent cell differentiation, flattening of cell shape during differentiation, secretion of lipids, desquamation, and the development of a spatial structure of the dermis due to cell division. By numerical simulations, we demonstrate that the model can produce a stable epidermis with well-organized layer structures. Extensive numerical investigations reveal that the stability of the layer structure depends on the supply rate of cells from the basal layer. Besides, by controlling the stiffness of the dermis, we show how the hardening of the dermis affects epidermal homeostasis. Finally, we show that our model can be used to simulate a skin disease that causes morphological changes in both the epidermis and the dermis, such as the corn (clavus). We also show an experimental result of the corn formation and compare it with the simulation result.

\section{Results}

\section{Maintenance of epidermal homeostasis}

We checked if the present model can exhibit a well-organized epidermal structure as a steady state. We performed simulations with two different conditions by changing the maximum number of cell divisions $N_{\text {div }}$ and the cell division period $T_{\mathrm{div}}$, which affects the cell supply rate from the basal layer.

When a sufficient number of cells are continuously supplied from the basal layer, a fully developed epidermis was formed with clearly separated layers, each consisting of spinous, granular, and cornified cells [Fig.1(a)], The thickness of each layer was fairly uniform in space and stable in time [Fig.1(a), (b); see also Fig.3]. Columnar structures of vertically stacked cells were also observed in the granular layer and the cornified layer [Fig.1(a), (b)]. The development of the epidermis was accompanied by the creation of upward protrusions in the initially flat dermis, with stem cells located at the tip of the protrusions. [Fig.1(b)]. We compared this result with another simulation in which the cell supply rate was reduced by choosing smaller $N_{\text {div }}$ and larger $T_{\text {div }}$. In this case, we observed not only a decrease of thickness, as expected, but also the destabilization of the layer structure [Fig.1(d)]. In particular, the boundary between the granular layer and the spinous layer became blurred due to isolated granular cells away from the bulk layer [Fig.1(e)]. The effect of reduced cell supply was also found in the lipid production: When the cell supply is sufficient, intercellular lipids were sufficiently released from cornified cells [Fig.1(c)]. When the cell supply was reduced, however, we observed insufficient lipid productions in a fraction of cells [Fig.1(f)]. These results suggest that our model can produce a stable epidermal structure when cell supply from the basal layer is sufficient and that the reduced cell supply could affect both structural stability and the internal cell dynamics like lipid production. 
(a)

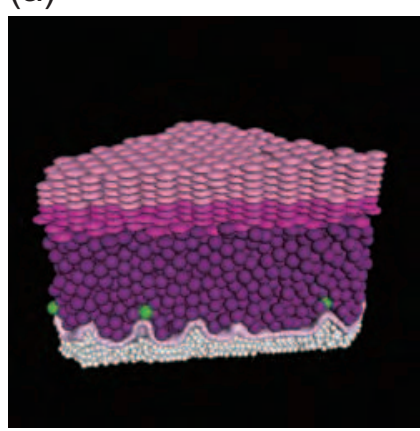

(d)

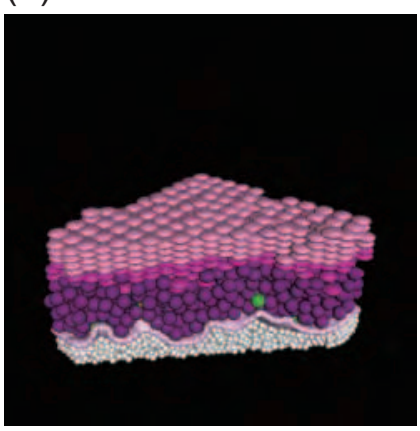

(b)

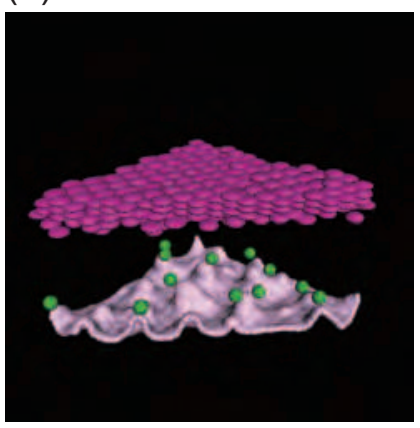

(e)

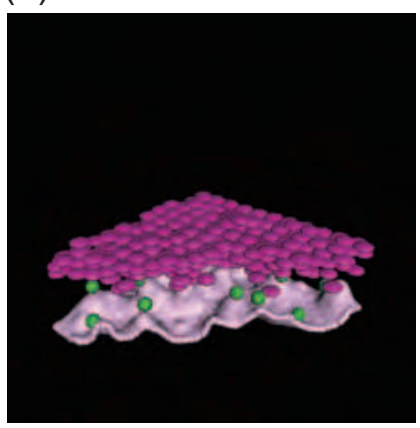

(c)

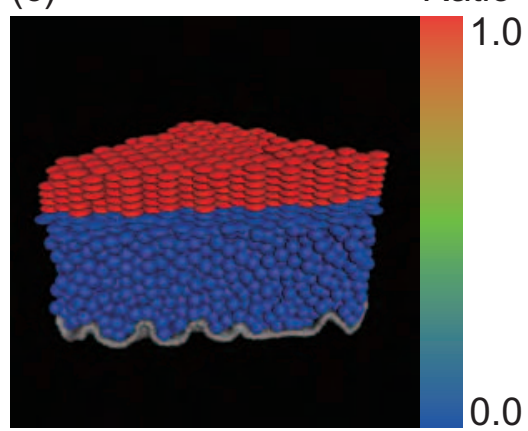

(f)

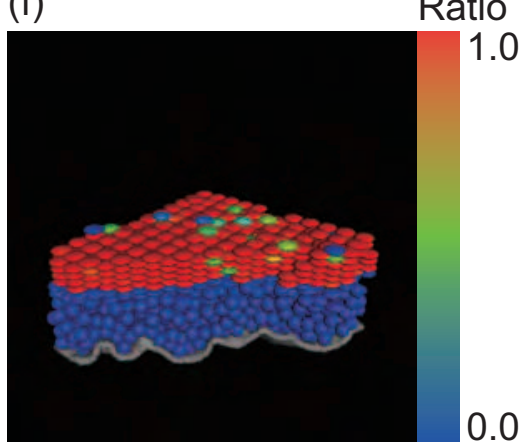

Figure 1. Simulation snapshots for two different sets of $N_{\text {div }}$ (maximum number of cell divisions) and $T_{\text {div }}$ (cell division period): (a-c) $\left(N_{\text {div }}, T_{\text {div }}\right)=(14,4.0)$, (d-f) $\left(N_{\text {div }}, T_{\text {div }}\right)=(8.0,4.4)$. (a) Overview of the epidermal model. From below: the dermis (white), the basement membrane (light pink), stem cells (green), basal and spinous cells (purple), granular cells (dark magenta), cornified cells (dark pink). (b,c) Same snapshots as (a): (b) The basement membrane, stem cells, and granular cells; (c) Ratio of the amount of lipids released from individual cells to the maximum lipid production. (d), (e), and (f) correspond with (a), (b), and (c), respectively. 


\section{Evaluation of epidermal conditions by changing cell supply rate}

Then we investigated the effect of the cell supply rate on the epidermal structure and the lipid production more systematically by varying the parameters $N_{\text {div }}$ and $T_{\text {div }}$. We focus on the granular and the cornified layers. To evaluate the structure of these layers, we introduce the following measures: For each cell layer (granular or cornified), we define the thickness $H$; the dispersion $G$, and the spatial variation of the thickness $E$. A schematic illustration of these measures is given in Fig.2(a) (Precise definitions are given in the Method): $H$ is the thickness of the bulk; $G$ expressed the largest vertical deviation of isolated cells from the bulk; and $E$ represents the magnitude of modulations of the bulk thickness. Different features can be captured by these measures: A cell layer with uniform thickness and a well-defined boundary with the adjacent cell layer should have small $G$ and $E$.

Figure 2 shows the evaluation measures as a function of $N_{\text {div }}$ vs $T_{\text {div }}$. In the granular layer, the parameter space can be divided into two regions [Fig.2(b)]: In the lower-right region, where the cell supply rate is large (with large $N_{\text {div }}$ and small $T_{\text {div }}$ ), the thickness $H$ is high and both the dispersion $G$ and the spatial variation $E$ (normalized by the thickness $H$ ) are small, indicating a spatially uniform, well-defined granular layer. On the other hand, in the upper-left region, where the cell supply rate is small (with small $N_{\text {div }}$ and large $T_{\text {div }}$ ), the opposite tendency is observed, with small $H$, large $G / H$, and large $E / H$, indicating a thin bulk layer with large spatial variation of the thickness and with many isolated cells from the bulk. The same tendency is seen in the cornified layer [Fig.2(c)]: in the lower-right region, the cornified layer also has large $H$ and small $G$ and $E$. We note, however, that the parameter region that produces large $G$ and $E$ values is narrower in the cornified layer than in the granular layer and that the magnitude of $G$ and $E$ is the larger in the granular layer. This suggests that the maintenance of the granular layer is more crucial for epidermal homeostasis.

In our model, barrier functions of the epidermis are estimated by the thickness and the amount of lipid production in the cornified layer. The same tendency is found also the lipid production: The mean amount of lipids released from individual cells (normalized by maximum lipid production) is high in the lower-right region and low in the upper-left region [Fig.2(e), ratio]. This is because the number of cells with inadequate lipid production increases when the cell supply reduces [Fig.2(e), count]. These results suggest that sufficient cell supply is required for lipid production, as well as structural stability.

\section{Effect of the stiffness of the dermis}

Next, to see how the structure and the barrier function would be affected by the deformability of the dermis, we performed a simulation by increasing the stiffness of the dermis and compared the result with the previous simulation used in Fig.1(a-c). By stiffening the dermis, we observed that the layer structure was worsened and lipid production was impaired [Fig.3(a-c)]. Significant differences were found especially in the granular layer [Fig.3(d)]: the thickness $H$ was greatly reduced, More isolated cells were observed (large $G / H$ ), and spatial variations were more enhanced (larger $E / H$ ). The differences were less significant but still recognizable in the cornified layer [Fig.3(e)]. Temporal fluctuations of these quantities were also enhanced, as indicated by error bars in Fig.3(d) and (e). Cornified cells with inadequate secretion of lipids was also found [Fig.3(f)].

The stiffening of the dermis directly affected the dermal shape, as diminished dermal undulations [Fig.3(b)]; a significant reduction was observed in the difference between the maximum and the minimum vertical displacements of the basement membrane [Fig.3(g)]. Since the diminished undulations reduce the surface area, fewer basal cells are accommodated by the basement membrane, which results in the reduction of the cell supply rate. These results suggest that the stiffening of the dermis disrupts the epidermal structure and barrier functions because of the reduction of the cell supply rate.

\section{Simulation of the formation of a corn}

We wondered if our mathematical modeling could simulate human diseases by adjusting parameters. A corn (also termed clavus) is a well-demarcated and painful callus and typically develops on the plantar skin where the repeated friction or pressure is applied. We hypothesized that modulation of one stem cell is sufficient for corn development. We performed a simulation with the following modifications: We selected one stem cell as an abnormal cell; Those cells produced from this abnormal stem cell would divide twice as fast in the basal layer and differentiate twice as 
(a)
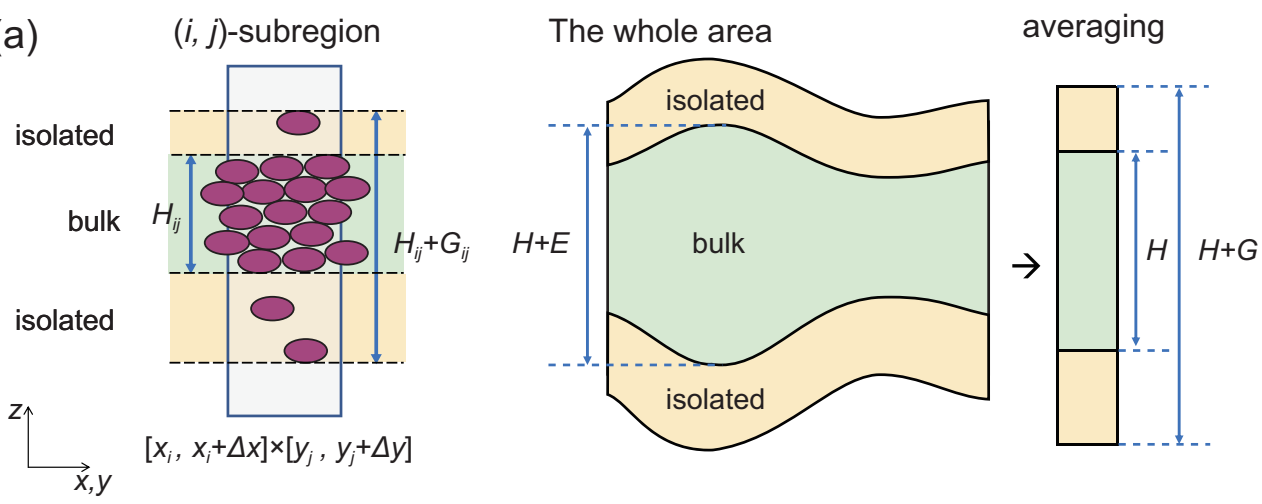

$\left[x_{i}, x_{i}+\Delta x\right] \times\left[y_{j}, y_{j}+\Delta y\right]$

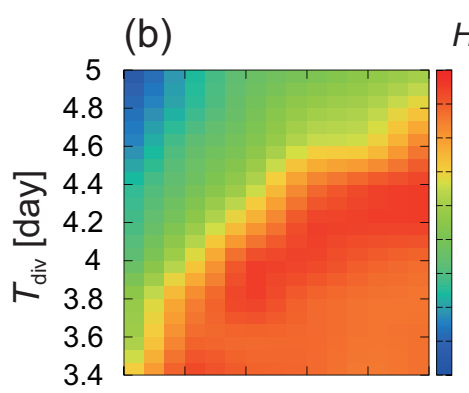

$H[\mu \mathrm{m}]$
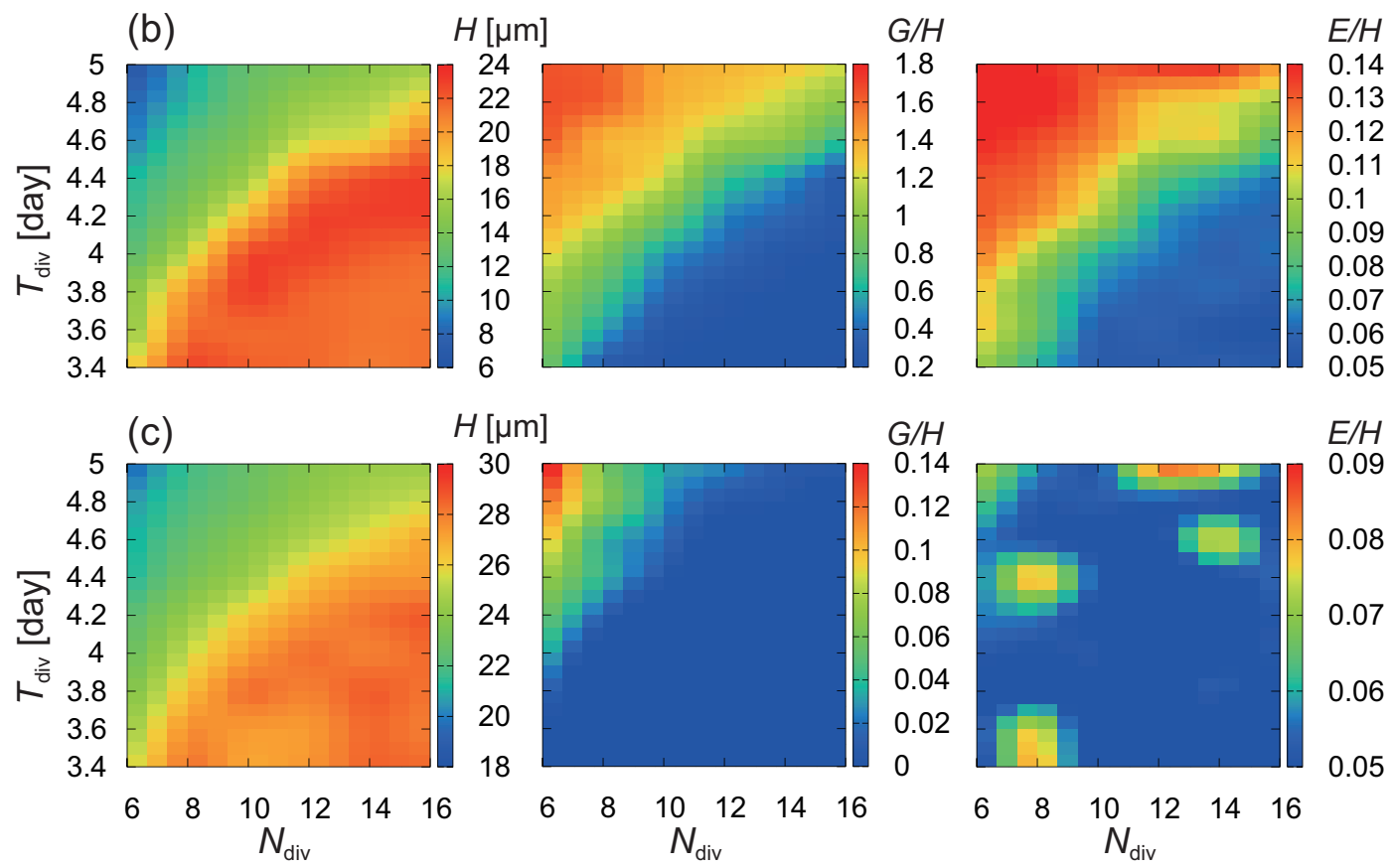

$H[\mu \mathrm{m}]$
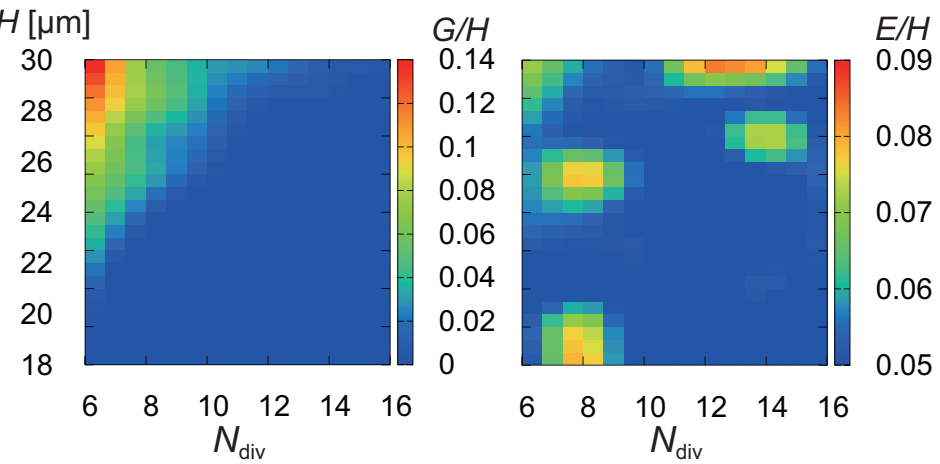

(d)

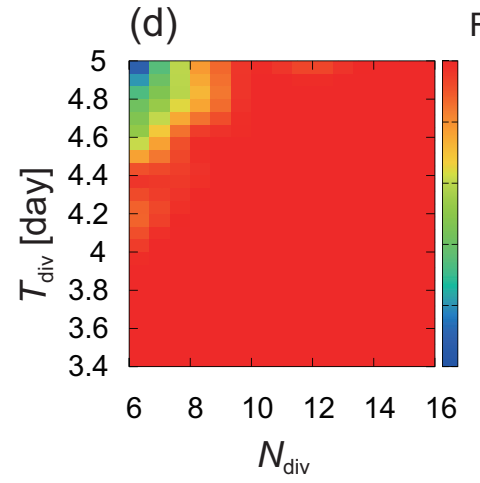

Ratio 1 0.98 0.96 0.94 0.92 0.9

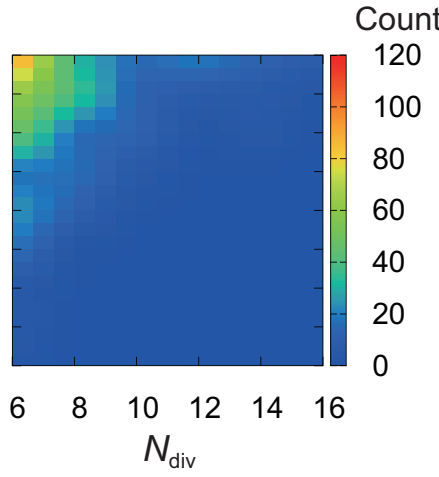

Figure 2. Evaluation of the epidermal structures and the lipid production as functions of $N_{\text {div }}$ (maximum number of cell divisions) and $T_{\text {div }}$ (cell division period). (a) Schematic illustration of the definition of the mean thickness $H$, the dispersion $G$, and the spatial variation $E$. (b) Thickness $H$, relative dispersion $G / H$ normalized by thickness, and relative spatial variation $E / H$ normalized by thickness for the granular layer. (c) $H, G / H$, and $E / H$ for the cornified layer. (d) Ratio of the amount of lipids released from cornified cells to the maximum lipid production. (e) Number of cornified cells with inadequate lipid production (less than $50 \%$ of the maximum). All values are time-averaged over 280 days (approximately 10 turnovers). See Methods for precise definitions of the evaluation functions. 
(a)

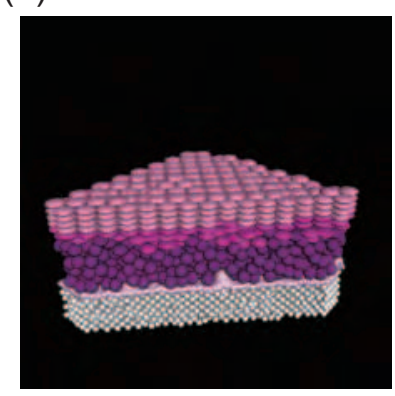

(d)

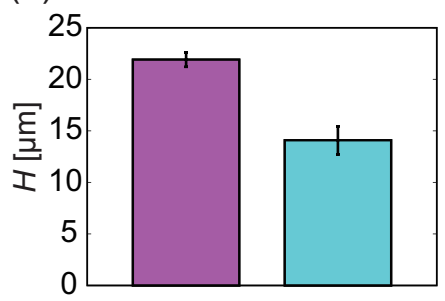

(e)

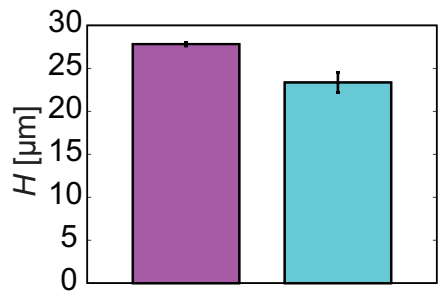

(f)

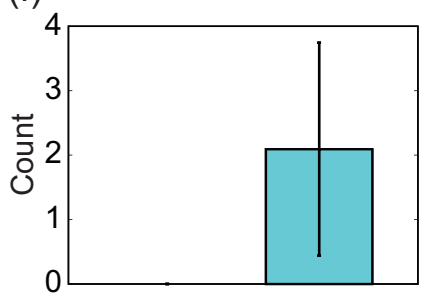

(b)
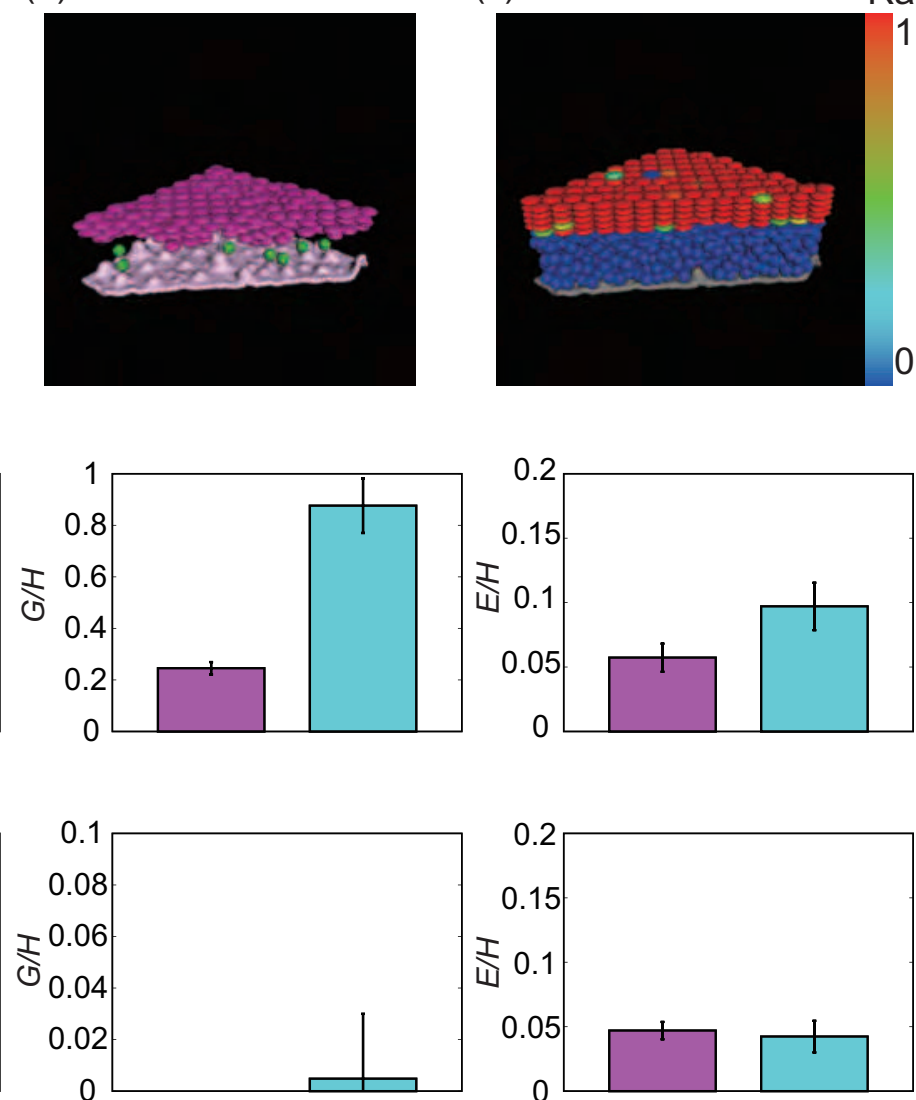

(g)

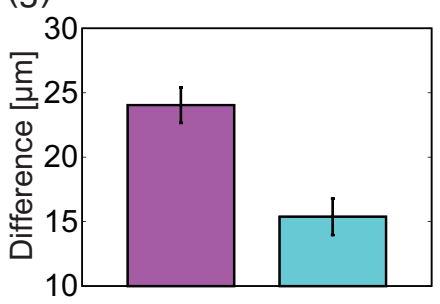

(c)

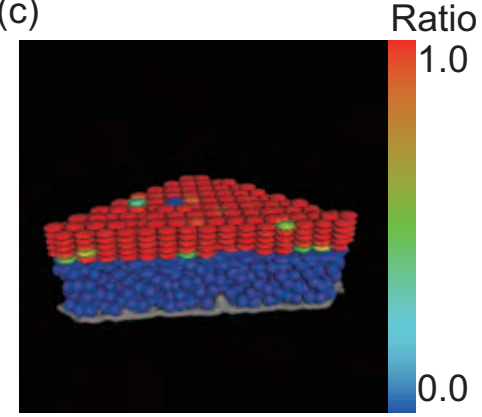

0

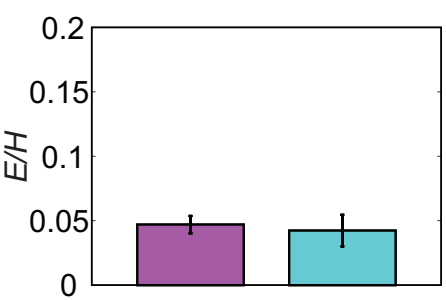

\section{: Normal dermis}

\section{: Stiffened dermis}

Figure 3. The effect of dermal stiffness. (a-c) Simulation with the stiffened dermis, presented in the same way as in Figs.1(a-c). (d-g) Comparison of the evaluation functions between the normal dermis and the stiffened dermis. (d) Evaluations of thickness $H$, normalized dispersion $G / H$, and normalized spatial variation $E / H$ for the granular layer. (e) $H, G / H$, and $E / H$ for the cornified layer. (f) Amplitude of the basement membrane deformation, defined by the difference between the maximum and minimum vertical displacements of the basement membrane. (g) Number of cells with inadequate lipid production (less than $50 \%$ of the maximum). All values are time-averaged over 280 days (approximately 10 turnovers). Error bars: standard deviations. 
fast in the suprabasal layer as those produced from a normal stem cell. The system size was made larger than the previous simulations. As shown in Fig.4(a), we found an inward intrusion of the cornified layer above the abnormal stem cell, where the intruding part of the cornified layer was mainly composed of cells produced by the abnormal stem cell [Fig.4(a), colored red]. In the basal layer, fast-dividing cells formed a cluster around the abnormal stem cell [Fig.4(b)], making a well-defined lesional area. Upward protrusions of the dermis were not observed in this lesional area, as in the non-lesional area, and the dermis in the lesional area was pushed downward, compared with the non-lesional area [Fig.4(c)].

(a)

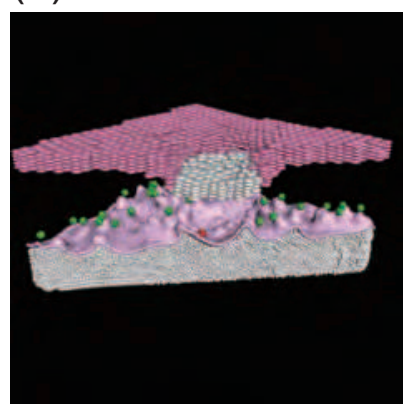

(b)

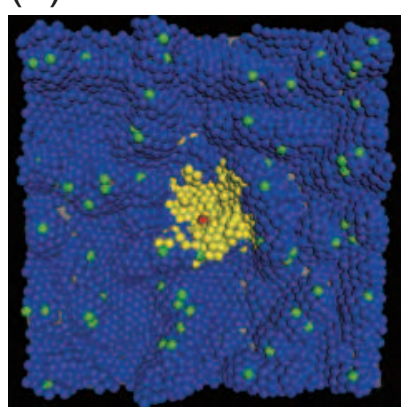

(c)

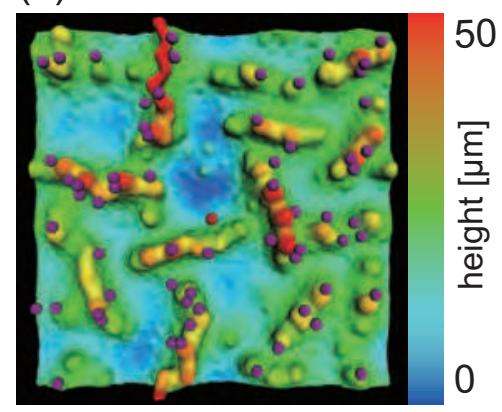

Figure 4. Simulation for the formation of a corn. (a) Cross section. One stem cell (red) has an abnormality among normal stem cells (green). White and pink cells are cornified cells produced by the abnormal stem cell and normal stem cells, respectively. (b) Top view of the basal layer. Yellow and blue cells are transit-amplifying (TA) cells that are originated from the abnormal stem cells and normal stem cells, respectively. (c) Top view of the basement membrane. The color indicates the vertical displacement of the baement membrane, measured from the lowest point. Stem cells are colored violet.

\section{Human corn morphology}

Then we asked if the human corn reproduces the dynamics of proliferation and differentiation seen in the mathematical model (Fig.4). We analyzed three corn specimens that were developed on the patients soles, and all of the samples shared similar findings. Hematoxylin and eosin (H\&E) staining showed a massive hyperkeratosis (thickening of the cornified layer) in the lesional area of the corn (Fig.5a), and the underlying epidermis was thinner than that of the non-lesional area (arrowheads). Parakeratosis (retention of the nuclei in the cornified layer) was also noted in the corn lesional area (Fig.5b), suggesting the premature differentiation in the corn epidermis. Keratin 6 (K6) has been known to show an alternate expression in the palmoplantar epidermis ${ }^{39}$ (Fig.5c, non-lesional area, white arrowheads), but this pattern was absent in the corn (Fig.5c, lesional area). Ki-67+ proliferative cells were more abundant in the corn than in the surrounding normal epidermis (Fig.5d). Besides, the epidermal differentiation markers (keratin 1 (K1) and 10 (K10) were absent, while the basal cell marker keratin 14 (K14) was retained even in the cornified layer in the corn (Fig.5e-g). This disturbed keratin pattern indicates that the corn epidermis does not have sufficient time to induce typical differentiation markers due to its fast differentiation. These data demonstrate that the mathematical model (Fig.4) recapitulates human corn morphology as well as epidermal hyperproliferation and rapid differentiation of the corn.

\section{Discussion}

The numerical results presented above are compatible with our previous results: Using the previous model with a flat, rigid dermis, we have already reported both the stable epidermal structure as in Fig. $1^{26}$ and the cell supply dependence of the stability of the suprabasal layer ${ }^{41}$. Also, the shape of the dermis, as well as the spatial distribution pattern of stem cells, is qualitatively the same as in the dermal deformation model ${ }^{29}$. These features are preserved in the present integrated model. The reduction of undulation magnitude in the dermis by stiffening is also consistent with the previous model ${ }^{29}$; but the present integrated model has also revealed that the stiffness could affect the 

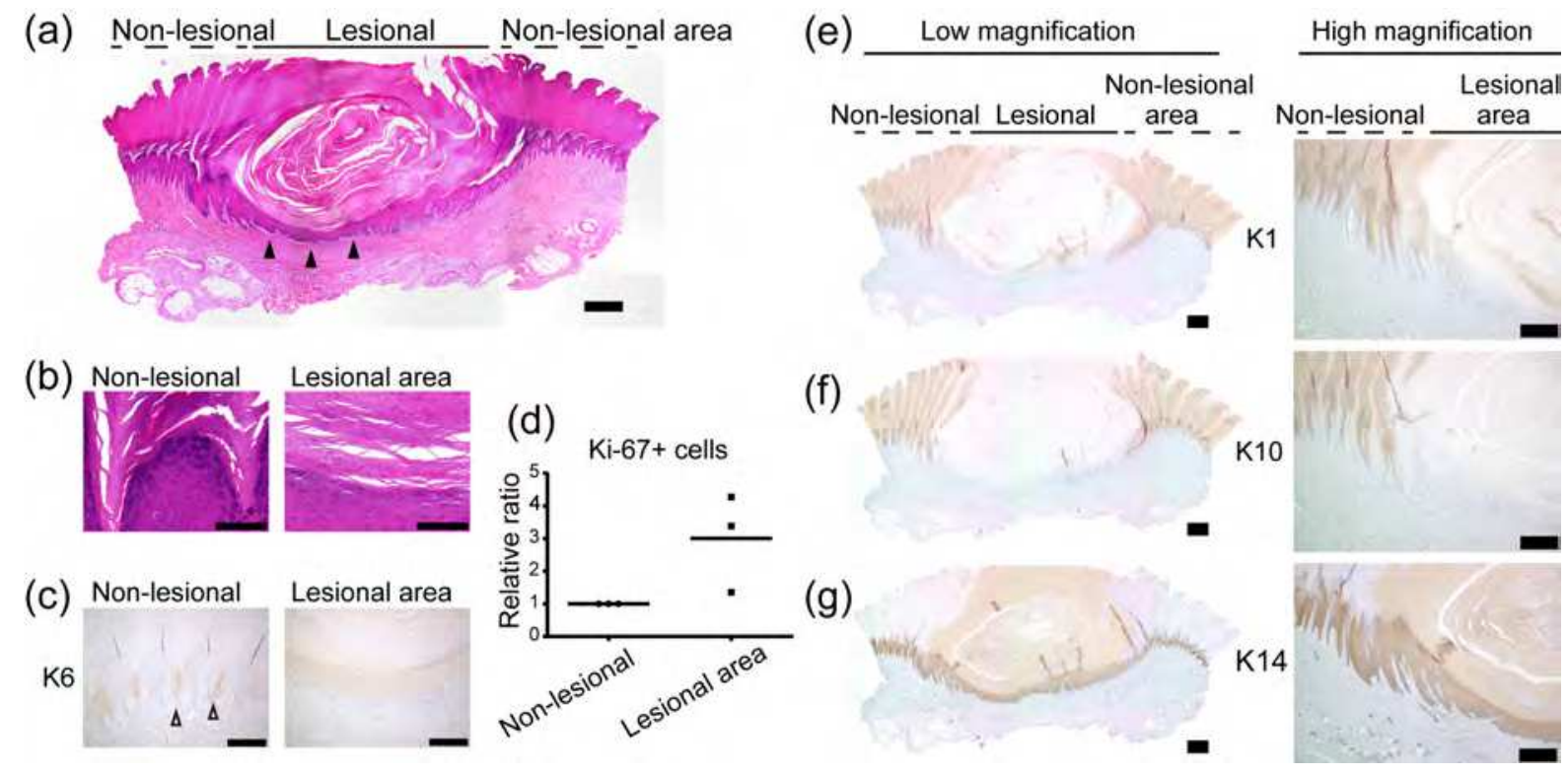
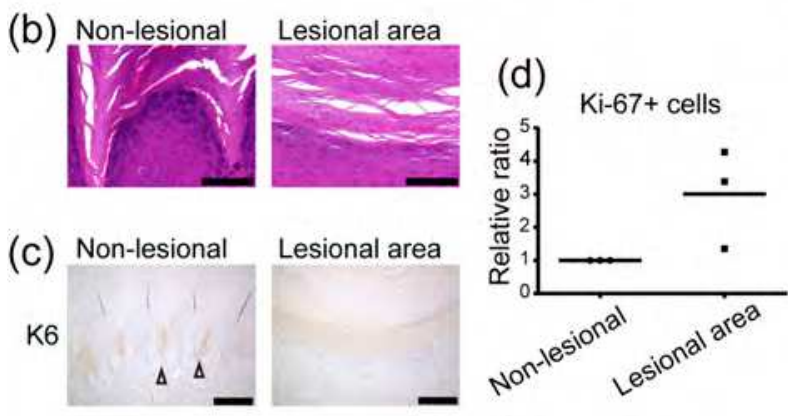

Figure 5. Histopathology of human corn (a, b) H\&E staining. Scale bar: $1 \mathrm{~mm}$ (a) and $100 \mu \mathrm{m}$ (b). (c) K6 labeling. Scale bar: $300 \mu \mathrm{m}$. (d) Quantification of Ki-67+ cells in the epidermis. (e-g) K1, K10, and K14 labeling. Scale bar: $1 \mathrm{~mm}$ (low magnification) and $500 \mu \mathrm{m}$ (high magnification).

suprabasal layers, as shown in Fig.3, which can be studied only by treating the epidermis and the deformable dermis simultaneously.

We have made two major modifications to the previous epidermal model ${ }^{26}$. First, the previous model did not consider the shape change of cells during differentiation. By taking this into account, we have succeeded in producing columnar structures in granular and cornified cells ${ }^{43,44}$ as shown in Fig.1, which was not found in the previous model. We note that the flattening process was also introduced in a different model ${ }^{5}$. Second, in addition to calcium ions, we have introduced a second factor that is released from cells undergoing cornification. In the previous model, we have shown that a localized layer of calcium ions is required to maintain a well-defined boundary between cornified cells and granular cells. This is achieved in the previous and the present models by the assumption that calcium ions released at the time of cornification accelerate cell differentiation, which causes stabilization of the boundary of the cell layer. How this mechanism works has been analytically confirmed by using a reaction-diffusion-advection model ${ }^{40}$. Since the previous model did not distinguish the granular and the spinous cells, however, an additional mechanism is needed to create another boundary between the granular and the spinous layers. Hence we introduced the second factor that can modify differentiation speed differently from calcium ions. The two factors work together to form two boundaries separating the spinous, granular, and cornified layers, as shown in Fig.1.

Our result on the stiffness of the dermis (Fig.3) may shed light on photoaging, a physiological senescence process induced by ultraviolet exposure: Ultraviolet destroys elastic fibers in the dermis, leading to dermal stiffness. Photoaging causes changes in the dermal structures, such as flattening of the basement membrane and thinning of the epidermis ${ }^{45}$, accompanied by the decrease in the number and the activity of stem cells ${ }^{46}$. This phenomenon is in accordance with our model, which predicts that, when the elasticity of the basement membrane is lost or the dermis is hardened by photoaging, undulations are suppressed and as a result, the thickness of the whole epidermis is reduced because of the diminishing surface area of the basement membrane accommodating fewer basal cells.

In summary, by combining the epidermal structure model ${ }^{26}$ and the dermal deformation model ${ }^{29}$, we have presented an integrated framework for simulating epidermal homeostasis. The previous model of epidermis ${ }^{26}$ had already found applications in simulating a thick epidermis equivalent on an undulating substrate ${ }^{28}$ or the epidermal proliferation under the reduced adhesion of progenitor cells to the basement membrane ${ }^{42}$. In this work, by consid- 
ering the deformation of the dermis, we could also simulate the formation of a corn. Our model is expected to be applied for simulating various diseases entailing structural changes of the dermis.

\section{Method}

\section{Histology of human samples}

H\&E staining and immunohistochemistry for formalin-fixed paraffin-embedded samples were performed on three human corn specimens. The following antibodies were used for immunohistochemistry: anti-K1 (ThermoFisher, Waltham, Massachusetts, USA; 34B4), anti-K6 (ThermoFisher; LHK6B), anti-K10 (Santa Cruz Biotechnology, Dallas, Texas, USA; LHP1), anti-K14 (ThermoFisher; LL002), and anti-Ki-67 (Santa Cruz Biotechnology; MIB1). Images of immunohistochemistry and $H \& E$ sections were captured with a BZ-9000 microscope (Keyence, Tokyo, Japan). For quantification of Ki-67+ cells in the epidermis, the whole areas of the specimens were analyzed using ImageJ (NIH, Bethesda, Maryland, USA). The number of Ki-67+ cells was normalized by the length of the epidermis in each section. The institutional review board of the Hokkaido University Graduate School of Medicine approved all human studies described above (ID: 14-063). The study was conducted according to the Declaration of Helsinki Principles. Participants provided written informed consent.

\section{The computational model}

A full description of the mathematical model is given in Supplementary Information. The overview is described below.

We consider the system composed of the dermis, the basement membrane, and the epidermis. The dermis is a soft elastic substrate, which is modeled by particles adhesive to each other. The stiffness of the dermis is controlled by modifying the adhesion strength. The basement membrane is modeled by particles connected in the form of a triangular lattice. The lattice edges are assigned stretching and bending energies so that the membrane exhibits elasticity. Membrane particles are adhesive to dermal particles. Epidermal cells are represented by spheroids, whose flattening rate depends on differentiation.

An epidermal cell is either a stem cell or transit-amplifying (TA) cell. Stem cells are strongly bound to the basement membrane, whereas TA cells are weakly bound. Cells passively move due to the pressure created by repeated cell division, which causes TA cells to leave the basement membrane. The basal layer is defined as a set of stem cells and TA cells that are bound to the basement membrane. Stem cells divide an infinite number of times, whereas TA cells (bound to the basement membrane) divide a finite number of times, $N_{\text {div }}$. Both cells follow a stochastic cell cycle, with the deterministic period $T_{\text {div }}$. TA cells not bound to the basement membrane are regarded as differentiated, which constitutes the suprabasal layer. Continuous cell division in the basal layer causes migration of cells towards the upper layers.

A cell is assigned a state variable; it starts to increase continuously when differentiated, with the increase accelerated by calcium ions and stimulants released by the cornified cells. Cell type changes as spinous, granular, or cornified in this order as the state variable increases. Lipids are produced inside granular cells and released when calcium ions increase, which typically occurs at cornification. After cornification, the cell peels off from the bulk (computationally, it is removed from the system), which is controlled by corneodesmosomes, cell-junction structures specific to cornified cells.

Hence, the mathematical model consists of equations of motion for dermal particles, membrane particles, and epidermal cells; cell division cycle; cell differentiation process; cell flattening during differentiation; lipid production; desquamation; and dynamics of calcium ions and stimulants. The scales of length and time in this model were determined so that the cell diameter is $10 \mu \mathrm{m}$ and the time-span between cornification and desquamation is 14 days, whereby the turnover time for the whole epidermis is approximately 28 days.

\section{Evaluation functions}

We define the measures $H, G$, and $E$ for the granular layer as follows (the same quantities are defined for the cornified layer in the same way). First, we divide the region $0 \leq x \leq L_{x}$ and $0 \leq y \leq L_{y}$ into $M_{1} \times M_{2}$ subregions, with $i, j$-subregions defined as $(i-1) \Delta_{x} \leq x<i \Delta_{x}$ and $(j-1) \Delta_{y} \leq y<j \Delta_{y}\left(i=1, \ldots, M_{1}, j=1, \ldots, M_{2}\right)$ with 
$\Delta_{x}=L_{x} / M_{1}$ and $\Delta_{y}=L_{y} / M_{2}$. Then we define the thickness $H_{i j}(t)$ in the $i, j$-subregion as the total (approximated) volume occupied by the cells divided by the area of the subregion:

$$
H_{i j}(t)=\frac{4 \pi R^{3} n_{i j}(t)}{3 \Delta_{x} \Delta_{y}},
$$

where $n_{i j}(t)$ is the number of granular cells. Also, we define the dispersion of cell distribution in the $z$ direction is defined as

$$
G_{i j}(t)=z_{i j}^{\max }(t)-z_{i j}^{\min }(t)-H_{i j}(t),
$$

where $z_{i j}^{\max }(t)$ and $z_{i j}^{\min }(t)$ are the maximum and the minimum $z$ value of granular cells in the $i, j$-subregion, respectively. The mean thickness $H$ and the dispersion $G(t)$ of the whole cell group are given by

$$
\begin{aligned}
& G(t)=\frac{1}{M_{1} M_{2}} \sum_{i=1}^{M_{1}} \sum_{j=1}^{M_{2}} G_{i j}(t), \\
& H(t)=\frac{1}{M_{1} M_{2}} \sum_{i=1}^{M_{1}} \sum_{j=1}^{M_{2}} H_{i j}(t) .
\end{aligned}
$$

The spatial modulation of the thickness $E$ is defined as the standard deviation of $H_{i j}$ :

$$
E(t)=\sqrt{\frac{1}{M_{1} M_{2}} \sum_{i=1}^{M_{1}} \sum_{j=1}^{M_{2}}\left(H(t)-H_{i j}(t)\right)^{2}} .
$$

\section{Acknowledgment}

This work was supported by CREST, JST (JPMJCR15D2), Maruho Takagi Dermatology Foundation, and Takeda Science Foundation.

\section{Author contributions}

M.N. and M.D. conceived and designed the project. M.N., Y.K., M.U. and T.G. formulated the mathematical model. K.O., Y.K., M.U. and T.G. developed simulation codes. K.O. performed the numerical simulations. H.K., M.W., K.N. performed the experiments. K.O., Y.K., K.N. and M.N. wrote the manuscript.

\section{Competing interests}

The authors declare no competing interests.

\section{References}

1. Elias, P. M. \& Feingold, K. R. Stratum corneum barrier function: definitions and broad concepts. In Skin Barrier (eds. Elias, P. M. \& Feingold, K. R.) 1-4 (Marcel Dekker, New York, 2005).

2. Natsuga, K. Epidermal barriers. Cold Spring Harb. Perspect. Med. 4, a018218 (2014).

3. Schaller, G. \& Meyer-Harmann, M. A modelling approach towards epidermal homoeostasis control. J. Theor. Biol. 247, 554-573 (2007).

4. X. Li et al. Skin Stem Cell Hypotheses and Long Term Clone Survival Explored Using Agent-based Modelling, Sci. Rep. 3, 1904 (2013).

5. Sütterlin, T., Tsingos, E., Bensaci, J., Stamatas, G. N. \& Grabe, N. A 3D self-organizing multicellular epidermis model of barrier formation and hydration with realistic cell morphology based on EPISIM. Sci. Rep. 7, 43472 (2017). 
6. Du, H., Wang, Y., Haensel, D., Lee, B., Dai, X. \& Nie, Q. Multiscale modeling of layer formation in epidermis, PLoS Comput. Biol. 14, e1006006 (2018).

7. Sun, T., Adra, S., Smallwood, R., Holcombe, M. \& MacNeil, S. Exploring hypotheses of the action of TGF- $\beta 1$ in epidermal wound healing using a 3D computational multiscale model of the human epidermis. PLoS ONE 4, e8515 (2009).

8. Adra, S., Sun, T., MacNeil, S., Holcombe, M. \& Smallwood, R. Development of a three dimensional multiscale computational model of the human epidermis. PLOS ONE 5, e8511 (2010).

9. Wang, Y. et al. A multiscale hybrid mathematical model of epidermaldermal interactions during skin wound healing. Exp. Dermatol. 28, 4 (2019): 493-502.

10. Zhang, $\mathrm{H}$. et al. Modelling epidermis homoeostasis and psoriasis pathogenesis. J. R. Soc. Interface 12, 20141071 (2015).

11. Sakuntabhai, A. et al. Mutations in $\mathrm{ATP}_{2} \mathrm{~A}_{2}$, encoding a $\mathrm{Ca}^{2+}$ pump, cause Darier disease. Nat. Genet. 21, 271-277 (1999).

12. $\mathrm{Hu}, \mathrm{Z}$. et al. Mutations in $\mathrm{ATP}_{2} \mathrm{C}_{1}$, encoding a calcium pump, cause Hailey-Hailey disease. Nat. Genet. 24, 61-65 (2000).

13. Meşe, G., Richard, G. \& White, T. W. Gap junctions: basic structure and function. J. Invest. Dermatol. 127, 2516-2524 (2007).

14. Forslind, B., Werner-Linde, Y., Lindberg, M. \& Pallon, J. Elemental analysis mirrors epidermal differentiation. Acta Derm. Venereol. 79, 12-17 (1999).

15. Mauro, T. et al. Acute barrier perturbation abolishes the $\mathrm{Ca}^{2+}$ and $\mathrm{K}^{+}$gradients in murine epidermis: quantitative measurement using PIXE. J. Invest. Dermatol. 111, 1198-1201 (1998).

16. Denda, M., Hosoi, J. \& Ashida, Y. Visual imaging of ion distribution in human epidermis, Biochem. Biophys. Res. Commun. 272, 789-795 (2000).

17. Menon, G. K., Price, L. F., Bommannan, B., Elias, P. M., \& Feingold, K. R. Selective obliteration of the epidermal calcium gradient leads to enhanced lamellar body secretion. J. Invest. Dermatol. 102, 789-795 (1994).

18. Elias, P. M. et al. Modulations in epidermal calcium regulate the expression of differentiation-specific markers. J. Invest. Dermatol. 119, 1128-1136 (2002).

19. Cornelissen, L. H., Oomens, C. W. J., Huyghe, J. M. \& Baaijenset F. P. T. Mechanisms that play a role in the maintenance of the calcium gradient in the epidermis. Skin Res. Tech. 13, 369-376 (2007).

20. Adams, M. P., Mallet, D. G. \& Pettet, G. J. Active regulation of the epidermal calcium profile. J. Theor. Biol. 301 112-121 (2012).

21. Kobayashi, et al. Mathematical modeling of calcium waves induced by mechanical stimulation in keratinocytes. PLOS ONE 9, e92650 (2014).

22. Grabe, N. \& Neuber, K. A multicellular systems biology model predicts epidermal morphology, kinetics and $\mathrm{Ca}^{2}+$ flow. Bioinformatics 21, 3541-3547 (2005).

23. Proksch, E., Brandner, J. M. \& Jensen J. M. The skin: an indispensable barrier. Exp. Dermatol. 17, 1063-1072 (2008).

24. Walker, D., Sun, T., MacNeil, S. \& Smallwood, R. Modeling the effect of exogeneous calcium on keratinocyte and $\mathrm{HaCat}$ cell proliferation and differentiation using an agent-based computational paradigm. Tissue Eng. 12, 23012309 (2006).

25. Sun, T. et al. An integrated systems biology approach to understanding the rules of keratinocyte colony formation. J. R. Soc. Interface 4, 10771092 (2007). 
26. Kobayashi, Y., Sawabu, Y., Kitahata, H., Denda, M. \& Nagayama, M. Mathematical model for calcium-assisted epidermal homeostasis. J. Theor. Biol. 397, 52-60 (2016).

27. Kobayashi, Y. \& Nagayama, M. Mathematical model of epidermal structure. In Applications + Practical Conceptualization + Mathematics = fruitful Innovation: Proceedings of the Forum of Mathematics for Industry 2014 (eds. Anderssen, R. S. et al.) 121-126 (Springer, Tokyo, 2016).

28. Kumamoto, J. et al. Mathematical-model-guided development of full-thickness epidermal equivalent. Sci. Rep. 8, 17999 (2018).

29. Kobayashi. Y. et al, Interplay between epidermal stem cell dynamics and dermal deformation. npj Comp. Mat. 4, 45 (2018).

30. Hannezo, E., Prost, J. \& Joanny, J. F. Theory of epithelial sheet morphology in three dimensions. Proc. Natl. Acad. Sci. U.S.A. 111, 27-32 (2014).

31. Basan, M., Joanny, J. F., Prost, J. \& Risler, T. Undulation instability of epithelial tissues. Phys. Rev. Lett. 106, 158101 (2011).

32. Tallinen, T. \& Biggins, J. S. Mechanics of invagination and folding: hybridized instabilities when one soft tissue grows on another. Phys. Rev. E 92, 022720 (2015).

33. Nelson, M. R., King, J. R. \& Jensen, O. E. Buckling of a growing tissue and the emergence of two-dimensional patterns. Math. Biosci. 246, 229-241 (2013).

34. Varner, V. D., Gleghorn, J. P., Miller, E., Radisky, D. C. \& Nelson, C. M. Mechanically patterning the embryonic airway epithelium. Proc. Natl. Acad. Sci. U.S.A. 112, 9230-9235 (2015).

35. Ben Amar, M. \& Jia, F. Anisotropic growth shapes intestinal tissues during embryogenesis, Proc. Natl. Acad. Sci. U.S.A. 110, 10525-10530 (2013).

36. Shyer, A. E. et al. Villification: how the gut gets its villi. Science 342, 212-218 (2013).

37. Dunn, S. J. et al. A two-dimensional model of the colonic crypt accounting for the role of the basement membrane and pericryptal fibroblast sheath. PLoS Comput. Biol. 8, e1002515 (2012).

38. Ciarletta, P. Buckling instability in growing tumor spheroids. Phys. Rev. Lett. 110, 158102 (2013).

39. Swensson, O. et al. Specialized keratin expression pattern in human ridged skin as an adaptation to high physical stress. Br. J. Dermatol. 139, 767-775 (1998).

40. Kobayashi, Y., Kitahata, H. \& Nagayama, M. Model for calcium-mediated reduction of structural fluctuations in epidermis. Phys. Rev. E 92, 022709 (2015).

41. Kobayashi, Y., Sawabu, Y., Ota, S. \& Nagayama, M. Mathematical model for epidermal homeostasis. In Mathematical Progress in Expressive Image Synthesis II (eds. Ochiai, H. \& Anjyo, K.) 119-123 (Springer, Tokyo, 2015).

42. Watanabe, M. et al. Type XVII collagen coordinates proliferation in the interfollicular epidermis. eLife 6, e26635 (2017).

43. MacKenzie, I. C. Ordered structure of the stratum corneum of mammalian skin. Nature 222, 881-882 (1969).

44. Christophers, E. Cellular architecture of the stratum corneum. J. Invest. Dermatol. 56, 165-169 (1971).

45. Fenske, N. A. \& Lober, C. W. Structural and functional changes of normal aging skin. J. Am. Acad. Dermatol. 15, 571 (1986).

46. Oh, J., Lee, Y. D. \& Wagers, A. J. Stem cell aging: mechanisms, regulators and therapeutic opportunities. Nat. Med. 20, 870 (2014). 


\section{Figures}

(a)

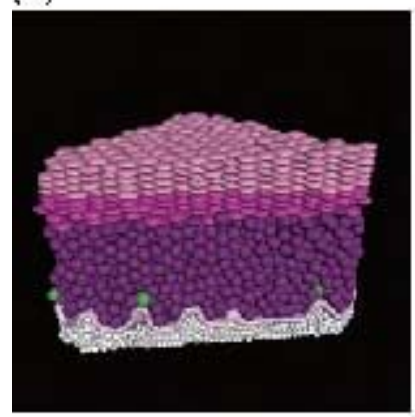

(d)

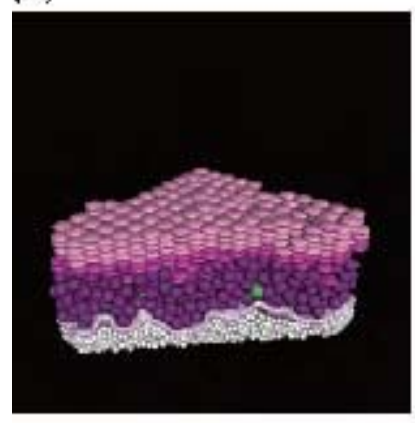

(b)

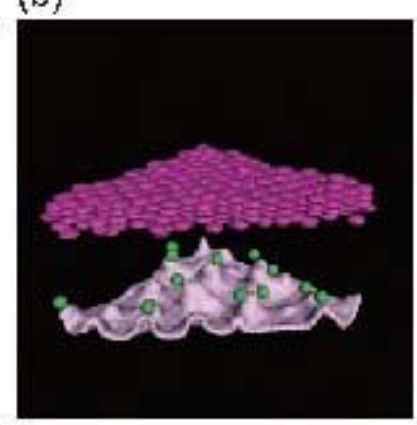

(e)

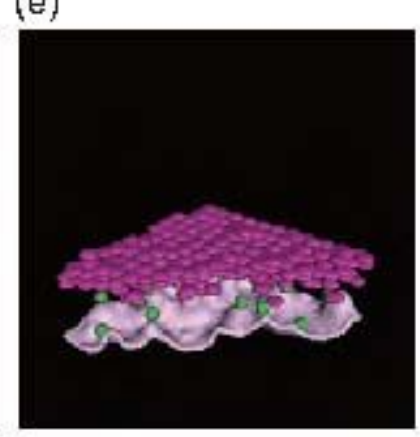

(c)

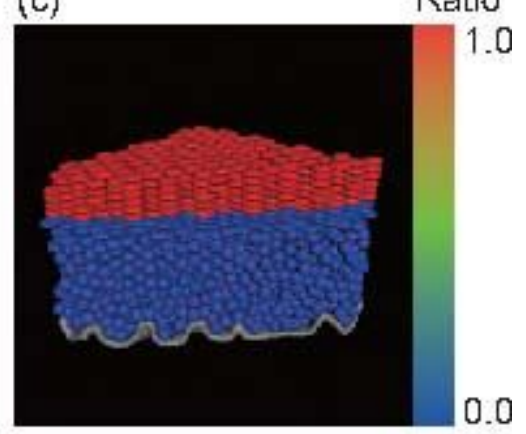

(f)

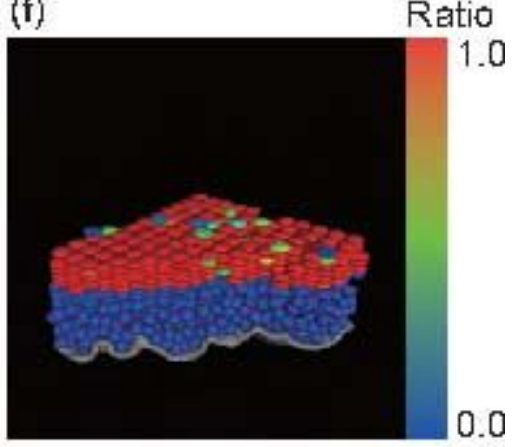

\section{Figure 1}

Simulation snapshots for two different sets of Ndiv (maximum number of cell divisions) and Tdiv (cell division period): $(\mathrm{a}-\mathrm{c})(\mathrm{Ndiv}, \mathrm{Tdiv})=(14,4 \cdot 0),(\mathrm{d}-\mathrm{f})($ Ndiv, Tdiv $)=(8 \cdot 0,4 \cdot 4)$. (a) Overview of the epidermal model. From below: the dermis (white), the basement membrane (light pink), stem cells (green), basal and spinous cells (purple), granular cells (dark magenta), cornified cells (dark pink). (b,c) Same snapshots as (a): (b) The basement membrane, stem cells, and granular cells; (c) Ratio of the amount of lipids released from individual cells to the maximum lipid production. (d), (e), and (f) correspond with (a), (b), and (c), respectively. 
(a)

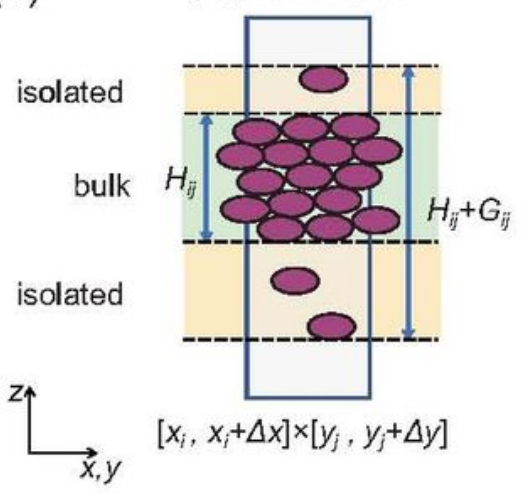

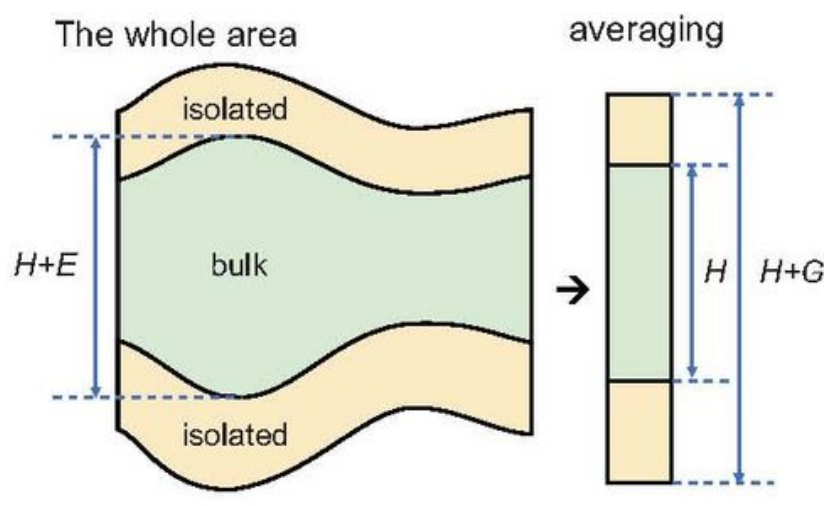
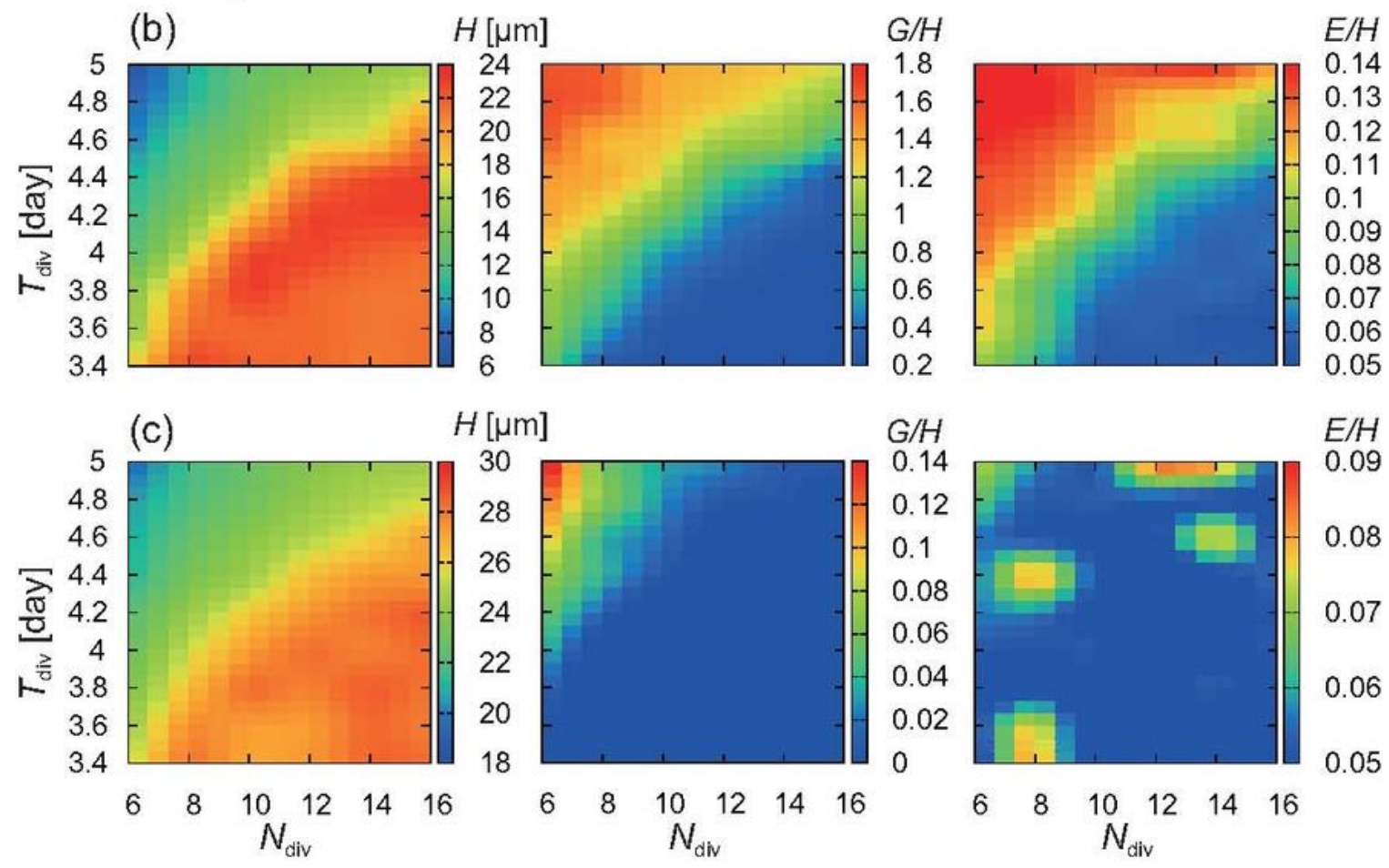

$H[\mu \mathrm{m}]$
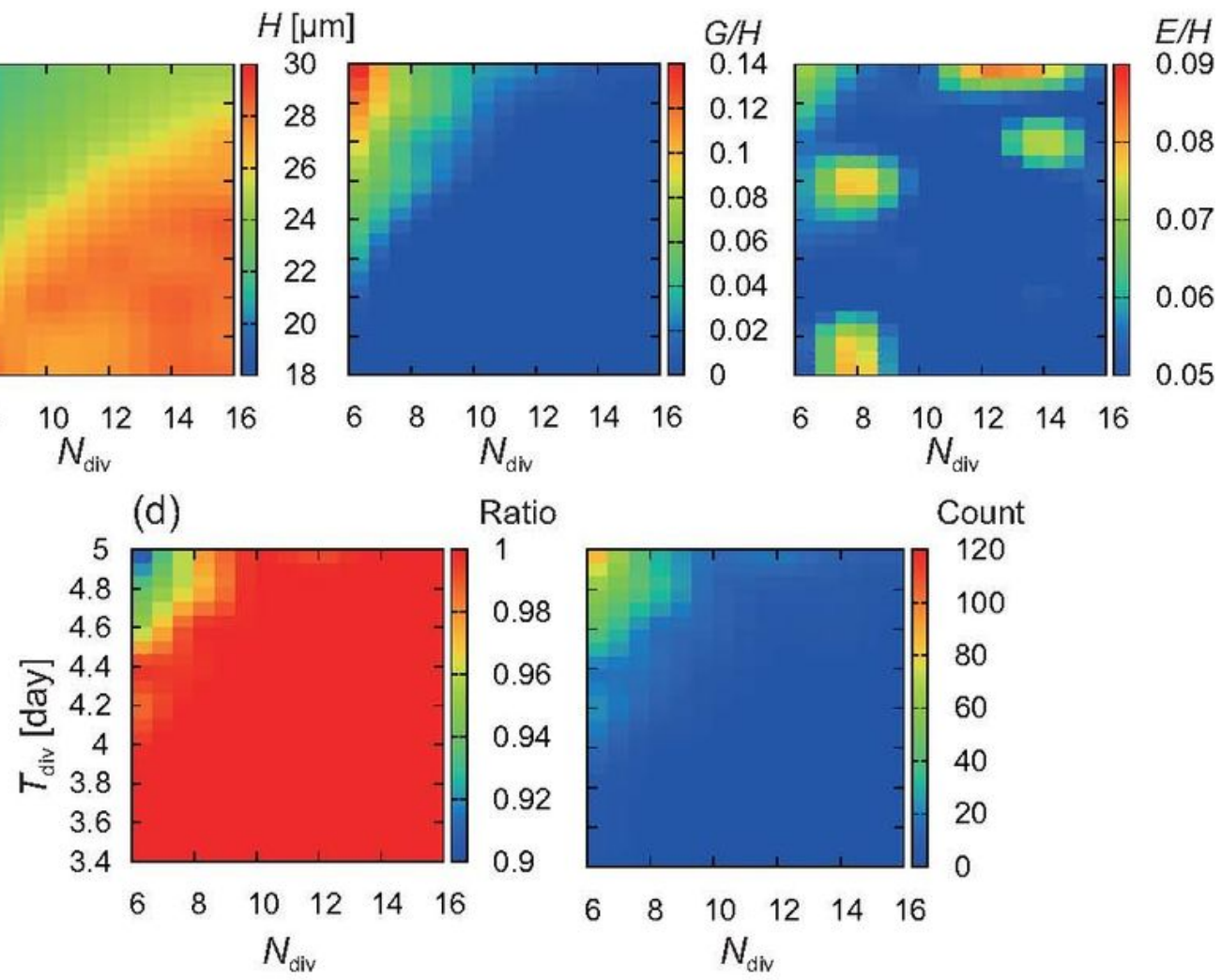

\section{Figure 2}

Evaluation of the epidermal structures and the lipid production as functions of Ndiv (maximum number of cell divisions) and Tdiv (cell division period). (a) Schematic illustration of the definition of the mean thickness $H$, the dispersion $G$, and the spatial variation $E$. (b) Thickness $H$, relative dispersion $G / H$ normalized by thickness, and relative spatial variation $\mathrm{E} / \mathrm{H}$ normalized by thickness for the granular layer. (c) $\mathrm{H}, \mathrm{G} / \mathrm{H}$, and $\mathrm{E} / \mathrm{H}$ for the cornified layer. (d) Ratio of the amount of lipids released from cornified cells to 
the maximum lipid production. (e) Number of cornified cells with inadequate lipid production (less than $50 \%$ of the maximum). All values are time-averaged over 280 days (approximately 10 turnovers). See Methods for precise definitions of the evaluation functions.

(a)

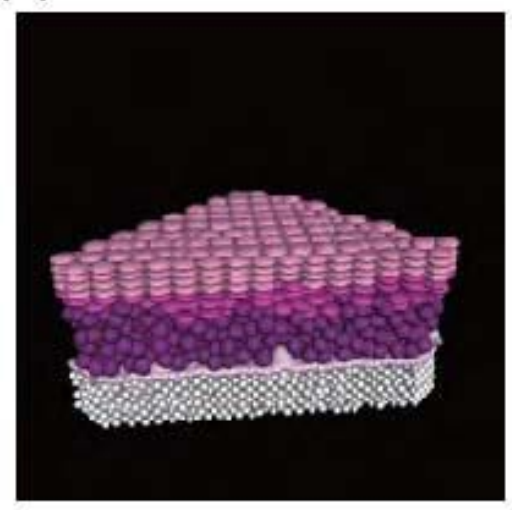

(d)

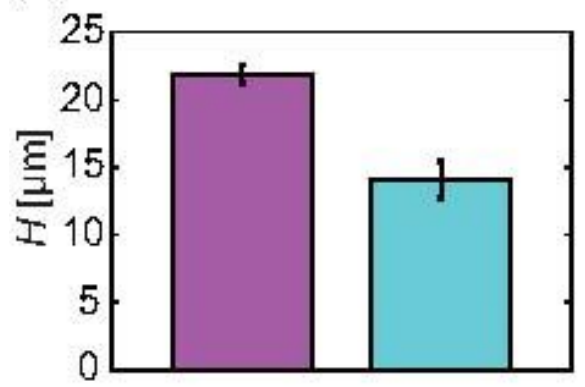

(e)

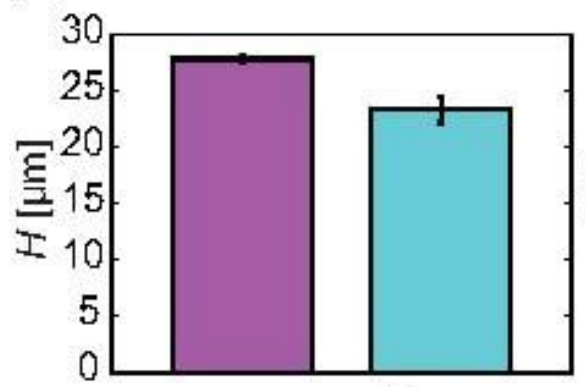

(f)

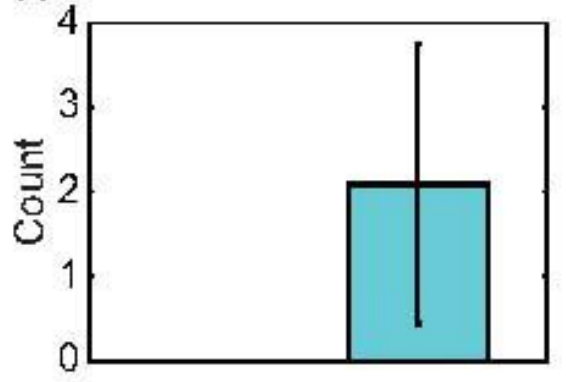

(c)
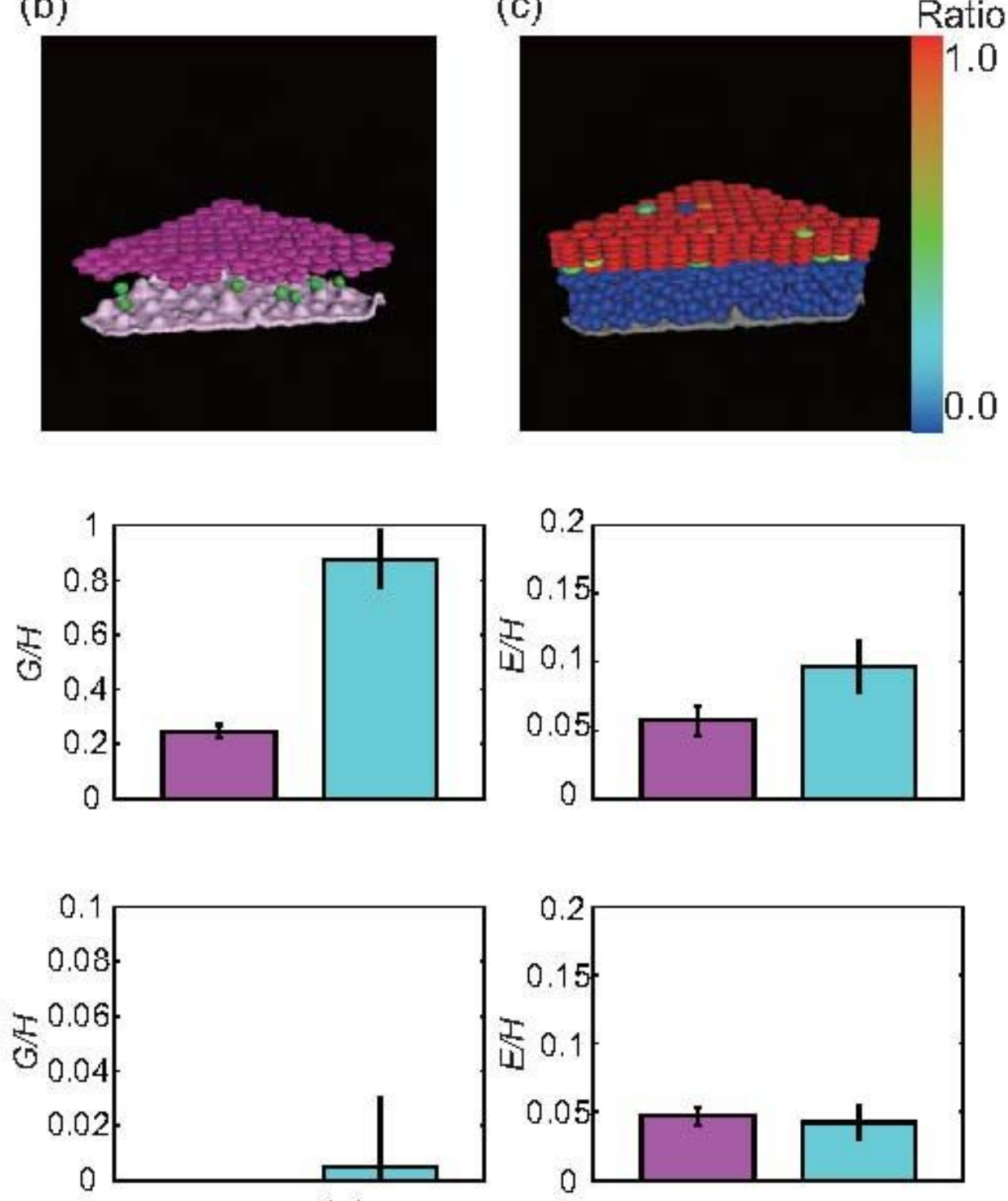

(g)

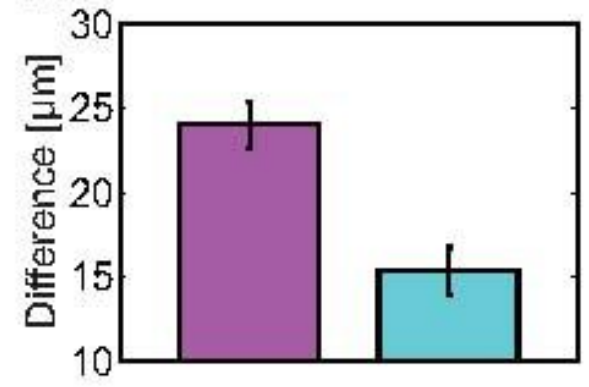

\section{: Nomal dermis} : Stiffened dermis

\section{Figure 3}

The effect of dermal stiffness. (a-c) Simulation with the stiffened dermis, presented in the same way as in Figs.1 (a-c). (d-g) Comparison of the evaluation functions between the normal dermis and the stiffened 
dermis. (d) Evaluations of thickness $\mathrm{H}$, normalized dispersion $\mathrm{G} / \mathrm{H}$, and normalized spatial variation $\mathrm{E} / \mathrm{H}$ for the granular layer. (e) $\mathrm{H}, \mathrm{G} / \mathrm{H}$, and $\mathrm{E} / \mathrm{H}$ for the cornified layer. (f) Amplitude of the basement membrane deformation, defined by the difference between the maximum and minimum vertical displacements of the basement membrane. (g) Number of cells with inadequate lipid production (less than $50 \%$ of the maximum). All values are time-averaged over 280 days (approximately 10 turnovers). Error bars: standard deviations.

\section{(a)}

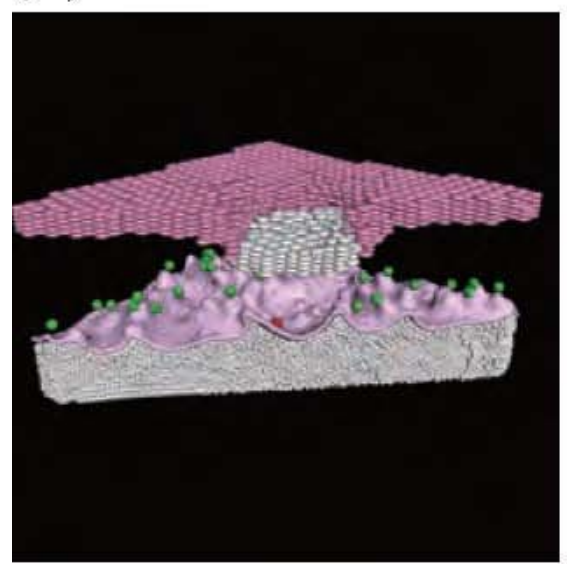

(b)

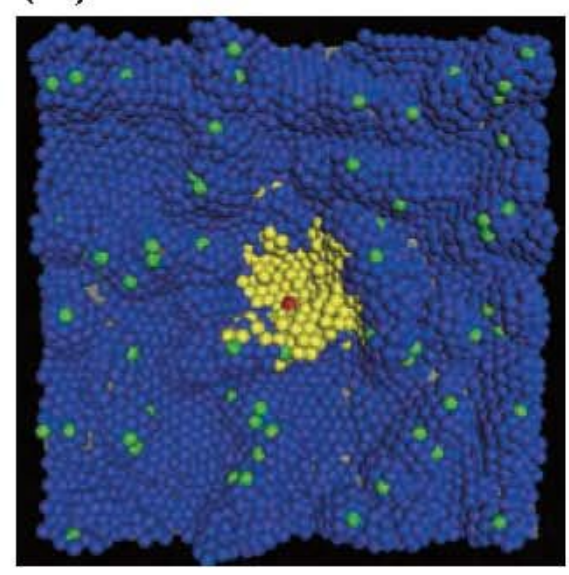

(c)

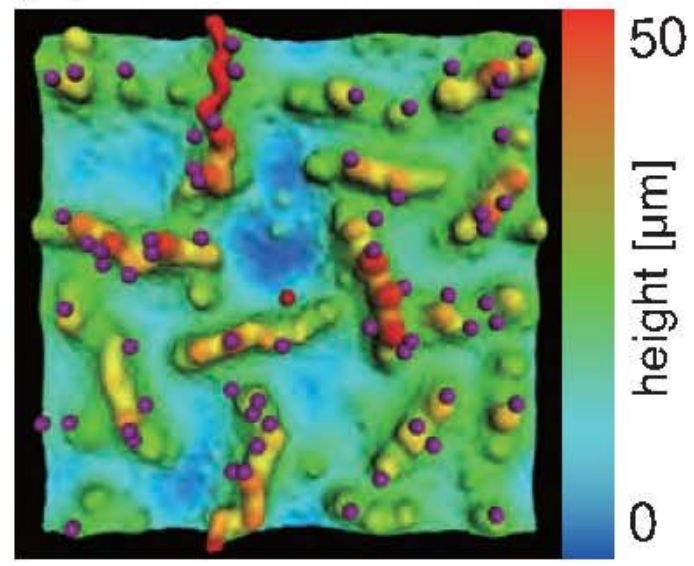

Figure 4

Simulation for the formation of a corn. (a) Cross section. One stem cell (red) has an abnormality among normal stem cells (green). White and pink cells are cornified cells produced by the abnormal stem cell and normal stem cells, respectively. (b) Top view of the basal layer. Yellow and blue cells are transitamplifying (TA) cells that are originated from the abnormal stem cells and normal stem cells, respectively. (c) Top view of the basement membrane. The color indicates the vertical displacement of the baement membrane, measured from the lowest point. Stem cells are colored violet.
(a)

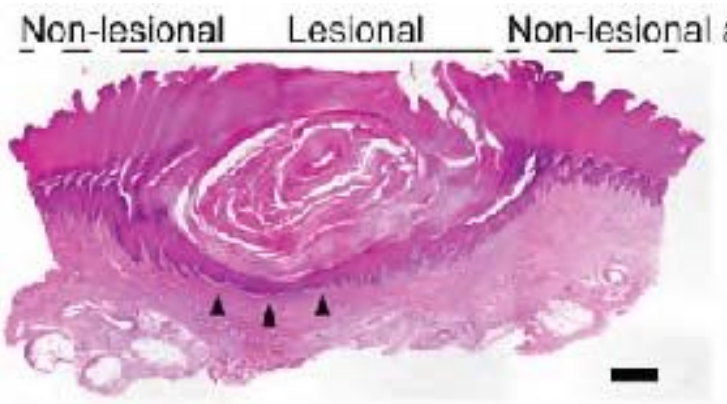
(e)
Low magnification area
Non-lesional
Non-lesional Lesiona
High magnification

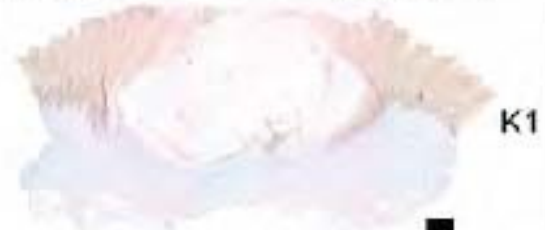
(f)
(b) Non-lesional

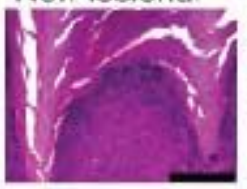
Lesional area

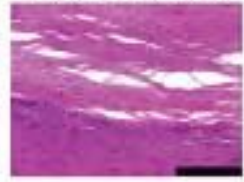
(c)

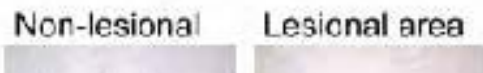
K6
$\triangle \Delta$
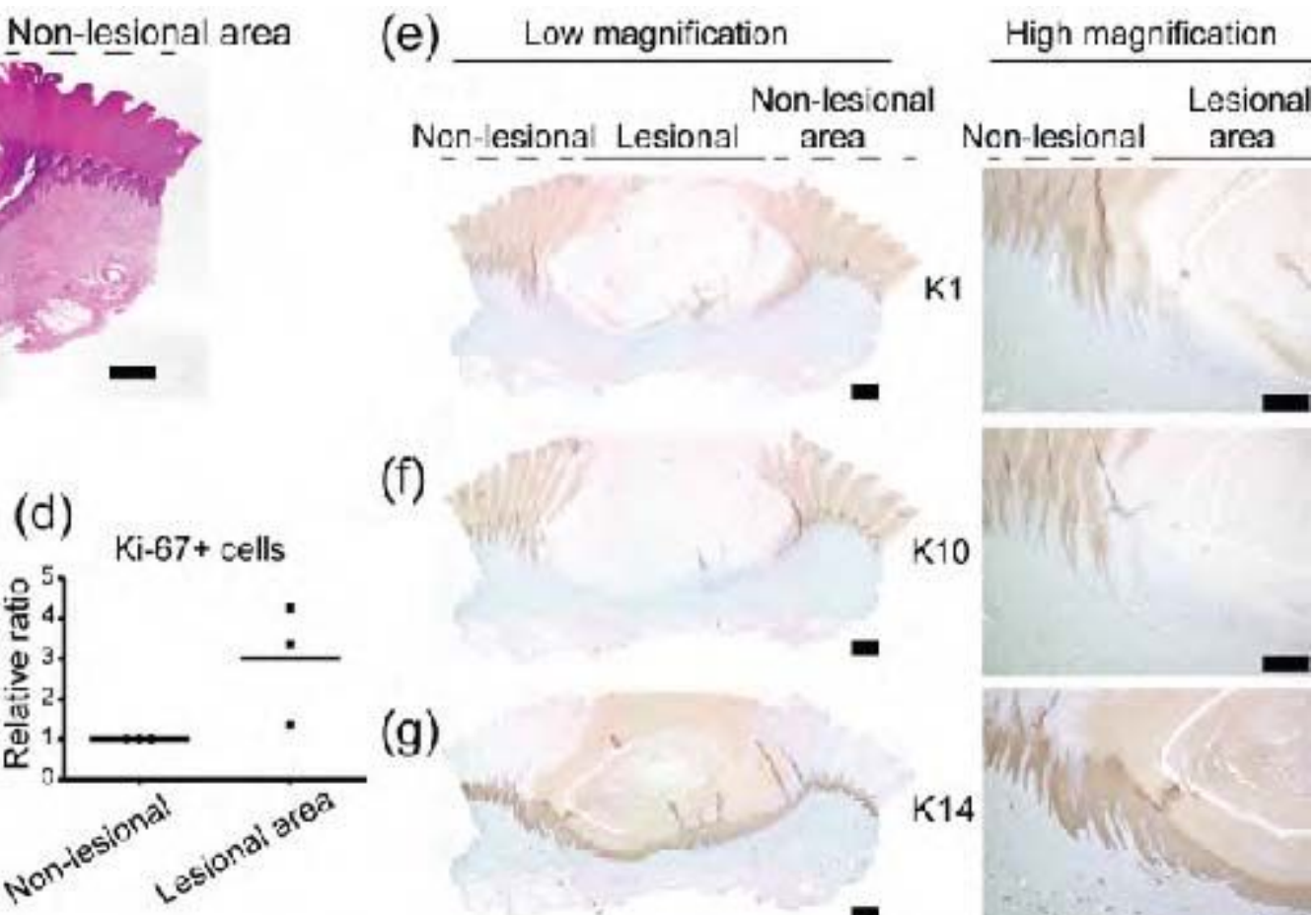
(g)

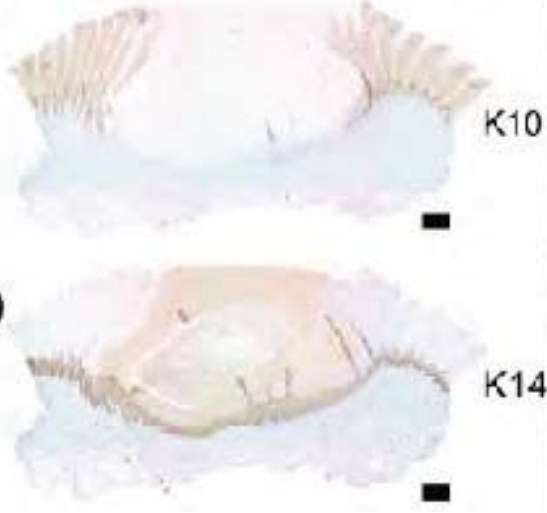
K14 


\section{Figure 5}

Histopathology of human corn (a, b) H\&E staining. Scale bar: $1 \mathrm{~mm}$ (a) and $100 \mu \mathrm{m}$ (b). (c) K6 labeling. Scale bar: $300 \mu \mathrm{m}$. (d) Quantification of Ki-67+ cells in the epidermis. (e-g) K1, K10, and K14 labeling. Scale bar: $1 \mathrm{~mm}$ (low magnification) and $500 \mu \mathrm{m}$ (high magnification).

\section{Supplementary Files}

This is a list of supplementary files associated with this preprint. Click to download.

- supplement.pdf 